\title{
Correlation of tumor relapse and elevated expression of survivin and vascular endothelial growth factor in superficial bladder transitional cell carcinoma
}

\author{
Y.W. Sun ${ }^{1,2}$, Q. Xuan ${ }^{2 *}$, Q.A. Shu ${ }^{2}$, S.S. Wu ${ }^{2}$, H. Chen ${ }^{2}$, J. Xiao ${ }^{2}$, P. Xiang ${ }^{2}$, \\ Y.P. $\mathrm{Zhu}^{2}$, F.L. Wang ${ }^{2}$ and S.T. Zhao ${ }^{1 *}$ \\ ${ }^{1}$ Department of Urology, Second Hospital of Shandong University, Jinan, \\ China \\ ${ }^{2}$ Department of Urology, Anhui Provincial Hospital, Hefei, China \\ *These authors contributed equally to this study. \\ Corresponding authors: S.T. Zhao / Q. Xuan \\ E-mail: 93873951@qq.com / xuanqxuanq76@hotmail.com
}

Genet. Mol. Res. 12 (2): 1045-1053 (2013)

Received August 23, 2012

Accepted February 13, 2013

Published April 2, 2013

DOI http://dx.doi.org/10.4238/2013.April.2.21

\begin{abstract}
Survivin and vascular endothelial growth factor (VEGF) are newly discovered tumor markers closely correlated with bladder cancer. We analyzed the expression of survivin and VEGF in paraffin-embedded tumor tissues from 78 patients with bladder transitional cell carcinoma (BTCC) using an immunohistochemistry method. Normal bladder mucosae from 10 non-BTCC cases were also included as a control group. All patients were closely followed up for tumor recurrence after undergoing transurethral resection of bladder tumor procedures. The positive expression rates of survivin and VEGF in superficial BTCC were 66.7\% (52/78) and 69.2\% (54/78), respectively, which were significantly higher than those in the control group, $0 \%(0 / 10)$. A positive correlation was found between survivin and VEGF expression $(\mathrm{r}=0.283, \mathrm{P}<0.01)$. Thirty-two of 78 patients $(41.0 \%)$ displayed recurrence during follow-up (median: 47; range:
\end{abstract}


7-62 months). The tumor recurrence rate in survivin $(+)$ patients was $53.8 \%(28 / 52)$, which was significantly higher than that in survivin(-) patients [15.4\% (4/26); $\mathrm{P}<0.05]$. The recurrence rate in $\operatorname{VEGF}(+) /$ VEGF(-) patients was 50.0\% (27/54) and 20.8\% (5/24), respectively (P $<0.05)$. The sensitivity for predicting the relapse of superficial BTCC was $87.5 \%$ in the survivin $(+)$ group, $84.4 \%$ in the VEGF $(+)$ group, and $78.1 \%$ in the survivin $(+) / \operatorname{VEGF}(+)$ group, and the specificity was $47.8,41.3$, and $65.2 \%$, respectively. Survivin and VEGF interact and jointly regulate the biological behavior of bladder cancer. Our results suggest that overexpression of survivin and VEGF accompany a higher risk of BTCC recurrence, making survivin and VEGF biomarkers for predicting the relapse of bladder cancer.

Key words: Survivin; Vascular endothelial growth factor; Recurrence; Bladder cancer

\section{INTRODUCTION}

Bladder cancer is the second most common malignancy of the urinary system, and bladder transitional cell carcinoma (BTCC) is the main histopathological type. Bladder cancer has a high recurrence rate, and no robust biomarker can currently predict tumor recurrence accurately. The ability to distinguish superficial tumors with adverse outcome from those unlikely to become invasive or clinically threatening would be of great clinical benefit. Superficial tumors with a more malignant phenotype may be better treated with early aggressive therapy. In recent years, many tumor markers have been found for determining the prognosis of bladder cancer as understanding of the molecular mechanisms of tumors has increased. However, few markers useful for predicting the relapse of bladder cancer are reported in the literature.

Survivin and vascular endothelial growth factor (VEGF) are newly discovered tumor markers closely correlated with bladder cancer. Survivin is a novel member of the inhibitor of apoptosis family. In addition to its anti-apoptotic function, survivin regulates cell division through proper assembly of the bipolar mitotic spindle and segregation of chromosomes (Deveraux et al., 1999). The overexpression of survivin has been reported in various human malignancies. Remarkably, increased survivin expression has been observed in the most common human neoplasms, including esophageal cancer (Grabowski et al., 2003), hepatocellular carcinoma (Ikeguchi et al., 2002), lung cancer (Monzo at al., 1999), ovarian cancer (Athanassiadou et al., 2008), leukemias (Mori et al., 2002), and neuroblastoma (Islam et al., 2000).

VEGF is the main growth stimulatory factor in tumor-related angiogenesis (Plate et al., 1992). A number of studies of various cancer types have confirmed that VEGF overexpression is closely correlated with metastasis, recurrence, and poor survival (Smith et al., 2000; Celen et al., 2004; Koukourakis et al., 2004; Jin et al., 2005). Therefore, searching for novel molecular biomarkers that can help clinicians improve prognosis and develop therapeutic interventions for BTCC patients is important. In this study, we assessed the expression of survivin and VEGF in BTCC and its potential value of predicting the recurrence of BTCC. 


\section{MATERIAL AND METHODS}

\section{Cases and clinical parameters}

We selected 78 patients with primary superficial BTCC who were treated in the Department of Urology, Anhui Provincial Hospital from October 2005 to September 2009. The patients included 61 males and 17 females with age ranging from 23 to 83 years (median age, 58.4 years). The tumor specimens from all patients were obtained during transurethral resection of bladder tumor (TUR-BT) procedures and were confirmed to be superficial BTCC by clinicians in the Department of Pathology, Anhui Provincial Hospital, China. Clinical stage determinations according to the Union for International Cancer Control tumor-node-metastasis criteria were as follows: $\mathrm{T}_{a}, 39$ patients; $\mathrm{T}_{1}, 39$ patients. Pathological grade assignments according to the World Health Organization classification system were the following: $G_{1}, 26$ patients; $G_{2}, 38$ patients; $G_{3}, 14$ patients. Patient recruitment and sample collection were performed within the guidelines of protocols approved by the institutional review boards of the respective hospitals. An additional 10 specimens from non-BTCC patients with normal bladder mucosa were obtained as a control group. Informed consent was obtained from all the patients who participated in this study.

All patients with superficial BTCC received intravesical instillation chemotherapy and they were closely followed after TUR-BT. Hydroxycamptothecin $(20 \mathrm{mg}$ integrated into $30 \mathrm{~mL}$ normal saline) was instilled into the bladder of patients without bladder perforation within the postoperative $24 \mathrm{~h}$ and into that of patients with bladder perforation 1 week after TUR-BT. Intravesical instillation then occurred once per week for 8 weeks and once per month for 18 months. Intravesical chemotherapy was stopped if the patient experienced a relapse. B-ultrasound, urine cytology, and cystoscopy were performed once every 3 months during the first 2 years after TUR-BT, then once every 6 months after the third postoperative year until the first instance of relapse.

\section{Immunohistochemistry}

Monoclonal antibody mouse anti-human survivin (Santa Cruz Inc., USA), rabbit anti-human VEGF polyclonal antibody (Santa Cruz), streptavidin-peroxidase, and 3,3'-diaminobenzidine chromogenic enzyme substrate kits (Zhongshan Biotechnology Company, China) were used for immunohistochemistry analysis. Briefly, paraffin-embedded tumor and control tissue specimens were sliced, dewaxed, and hydrated, incubated in retrieval buffer solution for antigen recovery, incubated with hydrogen peroxide for $10 \mathrm{~min}$, blocked with normal serum for $10 \mathrm{~min}$, and incubated with primary antibody for $60 \mathrm{~min}$. Finally, the slides were incubated with secondary antibody and Streptomyces antibiotic-peroxidase solution, stained with 3,3'-diaminobenzidine, and counterstained with hematoxylin, dehydrated, and mounted. Negative controls were prepared by substituting non-immune mouse or rabbit serum for the primary antibodies.

The immunostaining results were evaluated and scored independently by 2 pathologists who lacked knowledge of the clinicopathological outcomes of the patients. Digital images were manually scored according to staining intensity and morphology. Cells positive 
for survivin expression displayed brown-yellow granules in the cytoplasm. The staining intensity was graded as $0,1,2$, or 3 points for no yellow, buff, brown-yellow, and brown, respectively. Counts of the cells in 5 high-power fields observed randomly in each slide were scored as follows: 0 points for positive cells $<10 \%, 1$ point for $10-45 \%$ positive cells, 2 points for $46-65 \%$ positive cells, and 3 points for $>65 \%$ positive cells. Survivin expression was identified by the product of the scores of staining intensity multiplied by the scores of positive cells: the product was $<1$ for negative (-), 1-2 for weak positive $(+), 3-4$ for positive $(++)$, and 5-6 for strongly positive $(+++)$. Positive expression of VEGF was identified by the presence of brown-yellow granules in the cytoplasm. In 5 random high-power fields, a positive slide had $\geq 30 \%$ positive cells, and a negative slide had $<30 \%$ positive cells and no color staining.

\section{Statistical analysis}

Data were analyzed using the SPSS 12.0 software (SPSS Inc., Chicago, IL, USA). The associations between survivin, VEGF expression, and clinicopathological parameters were assessed using the chi-square test. A P value less than 0.05 was considered to be statistically significant.

\section{RESULTS}

All patients were followed for 7-62 months after TUR-BT. Median follow-up was 47 months. Bladder cancer recurrence confirmed with pathological biopsy occurred in 32 patients. The relapse rate was $41.0 \%$. The characteristics of the recurrent and non-recurrent groups are listed in Table 1.

Table 1. Characteristics of relapse and non-relapse groups.
\begin{tabular}{lcccccccc}
\hline Group & Gender (male/female) & Age (years) & \multicolumn{2}{c}{ Clinical stage } & & & \\
\cline { 4 - 8 } & & & $\mathrm{T}_{\mathrm{a}}$ & $\mathrm{T}_{1}$ & & $\mathrm{G}_{1}$ & $\mathrm{G}_{2}$ & $\mathrm{G}_{3}$ \\
\hline Relapse & $25 / 7$ & $60.1(25-78)$ & 12 & 20 & & 11 & 15 & 6 \\
Non-relapse & $36 / 10$ & $57.3(23-83)$ & 27 & 19 & & 15 & 23 & 8 \\
\hline
\end{tabular}

The expression patterns of survivin and VEGF staining are depicted in Figure 1. As shown in Table 2, the positive expression rates of survivin and VEGF were $0 \%(0 / 10)$ in normal bladder tissues, and 66.7 and $69.2 \%$, respectively, in superficial BTCC tissues. Expression of survivin and VEGF in BTCC was much higher than that in normal bladder tissues (both $\mathrm{P}$ $<0.01)$. A significant positive correlation was found between survivin and VEGF expression in BTCC tissues (Table 3).

The relapse rates of survivin(+) and survivin(-) patients were 53.8 and $15.4 \%$, respectively, and a significant difference was found between them $(\mathrm{P}<0.01)$. The recurrence rate of VEGF $(+)$ patients $(50.0 \%)$ was also significantly higher than that of VEGF(-) patients $(20.8 \%$; $\mathrm{P}<0.05$; Table 4). 




Figure 1. Immunohistochemistry staining of superficial bladder transitional cell carcinoma tissue. A. Positive staining of survivin was observed in bladder transitional cell carcinoma (BTCC); B. positive staining of VEGF was observed in BTCC; C. negative expression of survivin was observed in normal bladder tissue; D. negative expression of VEGF was observed in normal bladder tissue; E. negative control of BTCC.

Table 2. Expression of survivin and vascular endothelial growth factor (VEGF) in bladder transitional cell carcinoma (BTCC) and normal bladder tissues.

\begin{tabular}{lcccccc}
\hline Group & Total & \multicolumn{2}{c}{ Survivin } & P & & VEGF \\
\cline { 2 - 7 } & & $(+)$ & $(-)$ & & $(+)$ & $(-)$ \\
\hline Normal bladder & 10 & 0 & 10 & $<0.01$ & 0 & 10 \\
BTCC & 78 & 52 & 26 & & 54 & 24 \\
\hline
\end{tabular}

Table 3. Correlation between survivin and vascular endothelial growth factor (VEGF) expression in bladder transitional cell carcinoma.

\begin{tabular}{ccccc}
\hline & & Survivin & r & P \\
\cline { 2 - 5 } & $(+)$ & $(-)$ & & \\
\hline $\begin{array}{c}\text { VEGF } \\
(+)\end{array}$ & 41 & 13 & 0.283 & $<0.01$ \\
$(-)$ & 11 & 13 & & \\
\hline
\end{tabular}

Genetics and Molecular Research 12 (2): 1045-1053 (2013) 


\begin{tabular}{|c|c|c|c|c|}
\hline & Relapse & Non-relapse & Chi-square & $\mathrm{P}$ \\
\hline \multirow[t]{2}{*}{$\begin{array}{c}\text { Survivin } \\
(+)\end{array}$} & 28 & 24 & \multirow{3}{*}{10.598} & \multirow{3}{*}{$<0.01$} \\
\hline & 4 & & & \\
\hline \multirow{2}{*}{$\begin{array}{c}\text { VEGF } \\
(+)\end{array}$} & 4 & 22 & & \\
\hline & 27 & 27 & \multirow{2}{*}{5.842} & \multirow{2}{*}{$<0.05$} \\
\hline$(-)$ & 5 & 19 & & \\
\hline
\end{tabular}

The sensitivity and specificity of survivin expression for predicting BTCC relapse were 87.0 and $47.8 \%$, respectively. The sensitivity and specificity of VEGF expression for predicting BTCC relapse were 84.4 and $41.3 \%$, respectively. The sensitivity and specificity of the survivin and VEGF dual positivity for predicting BTCC relapse were 78.1 and $65.2 \%$, respectively.

\section{DISCUSSION}

Bladder cancer is a common tumor of the urinary system in which BTCC is the main pathological type, accounting for more than $90 \%$ of cases (Fleshner et al., 1996). Bladder cancer is classified as superficial bladder cancer $\left(T_{a}, T_{i}, T_{1}\right)$ or invasive bladder cancer $\left(T_{2}, T_{3}, T_{4}\right)$ according to the depth of tumor invasion into the bladder wall (Waters, 1996). Bladder cancer is characterized by a high recurrence rate, likely because of new tumor production, intraoperative shedding, planting of tumor cells, and other factors. Currently, TUR-BT remains the main treatment for superficial BTCC. Relapse occurs in 10-67\% of patients with superficial BTCC within 12 months after TUR-BT, and the recurrence rate reaches $24-84 \% 5$ years post-surgery (Sylvester et al., 2006). The probability of tumor invasion, metastasis, and deterioration increases after relapse, which leads to the effects of treatment means and poor prognosis. Therefore, indictors for effective assessment of recurrence risk are critical in superficial BTCC.

Survivin is a new member of the family of inhibitors of apoptosis proteins. Its molecular weight is the smallest, but it displays the strongest inhibition of apoptosis. Studies showed that survivin plays an important role in inhibiting cell apoptosis, regulating cell mitosis, and participating in the formation of new blood vessels (Zhu et al., 2005). Survivin blocks cell apoptosis by specifically binding to caspase- 3 and caspase-7, which are located in the mitotic spindle microtubules, and inhibiting their activity during mitosis (Suzuki et al., 2000). The distribution of survivin expression has obvious selection in tissues and cells. Expression is absent in cells of mature tissue, and traces of expression are found in the thymus and gonads, for example. Otherwise, significant survivin expression occurs in a variety of human malignant tumors such as bladder, stomach, lung, and breast (Rohayem et al., 2000). In this study, we found that the rate of positive survivin expression was $66.7 \%$ in patients with superficial BTCC. This rate was significantly higher than that $(0 \%)$ in normal controls $(\mathrm{P}<0.01)$ and is consistent with that mentioned in literature reports (Swana et al., 1999).

Tumor occurrence involves not only abnormal differentiation and proliferation of cells but also inhibition of cell apoptosis. Suppression of apoptosis allows mutant cells to survive, ultimately leading to tumor development over time (Kirkali et al., 2005). The elevated 
expression of survivin in tumor tissue may be regulated by nuclear factor-kappaB, protein kinase B, signal transducer and activator of transcription 3, and other factors. The overexpression of survivin increases its anti-apoptotic role in mitosis, thereby promoting tumor growth and metastasis. Therefore, it has great significance in early diagnosis, predicting relapse and prognosis in bladder cancer. Some researchers have investigated the relationship among survivin mRNA expression in tumor tissues, bladder washings of patients with superficial BTCC, and recurrence and concluded that high survivin mRNA expression indicates early relapse in bladder cancer (Schultz et al., 2003, 2004). Our study found that the relapse rate of survivin $(+)$ patients was significantly higher than that of survivin $(-)$ patients $(\mathrm{P}<0.05)$. Our results suggested that positive expression of survivin accompanies a higher risk of relapse in superficial BTCC. Survivin may become an index for predicting the relapse of bladder cancer during the follow-up period.

VEGF is a highly specific factor for promoting vascular endothelial cell division and inducing angiogenesis. VEGF is also a potent angiogenic factor as a multifunctional cytokine. VEGF is found in normal human tissues such as heart, lung, kidney, and bladder. However, VEGF expression in normal tissue occurs only at the low levels necessary to maintain normal blood vessel density and basic penetration functions for facilitating the transport of nutrients. Since VEGF is secreted by tumor cells, a variety of malignant tumors, including bladder cancer, show significant VEGF expression. In this study, the positive expression rate of VEGF in superficial BTCC was $69.2 \%$, which is significantly higher than that in normal bladder tissues $(\mathrm{P}<0.01)$. VEGF stimulates vascular endothelial cell proliferation and migration and promotes angiogenesis. Angiogenesis is necessary for tumor growth, invasion, relapse, and metastasis. Increased expression of VEGF can promote tumor angiogenesis, which supplies oxygen and nutrients for tumor cell growth and rapid proliferation leading to malignant progression.

Sato et al. (1998) found that positive expression of VEGF in patients with recurrent BTCC is significantly higher than that of VEGF in patients without recurrence within 2 postoperative years. The authors confirmed that VEGF can be regarded as an index for assessing malignancy, invasion, and recurrence of bladder cancer. The results of our study show that the recurrence rate of patients with $\operatorname{VEGF}(+)$ expression was significantly higher than that of patients with VEGF(-) expression $(\mathrm{P}<0.05)$, indicating that abnormal VEGF expression is closely related to the recurrence of bladder cancer and is a useful indicator for predicting BTCC relapse.

Recent studies have shown that apoptosis inhibition is closely correlated with angiogenesis in the occurrence, development, invasion, recurrence, and metastasis of tumors. The process of apoptosis is regulated not only by survivin but also by VEGF and the process of angiogenesis (Riedel et al., 2001). The positive expression of VEGF in tumor angiogenesis in endothelial cells promotes and induces high expression of survivin, which then upregulates the expression of angiopoietin-1, a critical factor for maintaining vascular stability and lumen formation during the course of angiogenesis (Tran et al., 1999). In this study, the expression of survivin was positively correlated with that of VEGF in superficial BTCC tissues $(r=0.283$, $\mathrm{P}<0.01$ ). The inhibition of apoptosis by survivin and the stimulation of tumor angiogenesis by VEGF are interlinked, and they influence each other through signal transduction networks, collaboratively regulating the biological behavior of bladder cancer.

These results indicated that co-analysis of VEGF and survivin protein expression in superficial BTCC tissues is valuable for patient prognosis evaluation. In this study, the sen- 
sitivities of survivin and VEGF for predicting the relapse of superficial BTCC were 87.5 and $84.4 \%$, respectively, and the specificities were 47.8 and $41.3 \%$, respectively. The sensitivities and specificities for predicting the relapse of superficial BTCC were 78.1 and $65.2 \%$, respectively, in patients with dual-positive survivin and VEGF expression.

The present data show that abnormal survivin and VEGF expression has important value for predicting the relapse of bladder cancer, and a combined analysis of both parameters may be especially informative. Finding relevant tumor markers for evaluating the prognosis of bladder cancer is necessary, because the occurrence and development of bladder cancer is a multi-stage, multi-factor, and multi-gene synergistic event.

Currently, clinical grade and tumor-node-metastasis stage for bladder cancer are insufficient to predict prognosis. Patients at the same clinical stage and grade often show different clinical courses. This study demonstrates that the expression of survivin is correlated with VEGF expression in superficial BTCC. Moreover, survivin and VEGF are both closely correlated with poor prognosis in BTCC. We suggest that detection of survivin and VEGF expression might help predict the prognosis and possibility of recurrence in patients with BTCC.

\section{REFERENCES}

Athanassiadou P, Grapsa D, Athanassiades P, Gonidi M, et al. (2008). The prognostic significance of COX-2 and survivin expression in ovarian cancer. Pathol. Res. Pract. 204: 241-249.

Celen O, Kahraman I, Yildirim E and Berberoglu U (2004). Correlation of vascular endothelial growth factor (VEGF) and CEA with clinicopathological variables in colorectal cancer patients. Neoplasma 51: 293-299.

Deveraux QL and Reed JC (1999). IAP family proteins - suppressors of apoptosis. Genes Dev. 13: 239-252.

Fleshner NE, Herr HW, Stewart AK, Murphy GP, et al. (1996). The National Cancer Data Base report on bladder carcinoma. The American College of Surgeons Commission on Cancer and the American Cancer Society. Cancer 78: $1505-1513$

Grabowski P, Kuhnel T, Muhr-Wilkenshoff F, Heine B, et al. (2003). Prognostic value of nuclear survivin expression in oesophageal squamous cell carcinoma. Br. J. Cancer 88: 115-119.

Ikeguchi M, Ueda T, Sakatani T, Hirooka Y, et al. (2002). Expression of survivin messenger RNA correlates with poor prognosis in patients with hepatocellular carcinoma. Diagn. Mol. Pathol. 11: 33-40.

Islam A, Kageyama H, Takada N, Kawamoto T, et al. (2000). High expression of Survivin, mapped to 17q25, is significantly associated with poor prognostic factors and promotes cell survival in human neuroblastoma. Oncogene 19: 617-623.

Jin Q, Hemminki K, Enquist K, Lenner P, et al. (2005). Vascular endothelial growth factor polymorphisms in relation to breast cancer development and prognosis. Clin. Cancer Res. 11: 3647-3653.

Kirkali Z, Chan T, Manoharan M, Algaba F, et al. (2005). Bladder cancer: epidemiology, staging and grading, and diagnosis. Urology 66: 4-34.

Koukourakis MI, Papazoglou D, Giatromanolaki A, Bougioukas G, et al. (2004). VEGF gene sequence variation defines VEGF gene expression status and angiogenic activity in non-small cell lung cancer. Lung Cancer 46: 293-298.

Monzo M, Rosell R, Felip E, Astudillo J, et al. (1999). A novel anti-apoptosis gene: Re-expression of survivin messenger RNA as a prognosis marker in non-small-cell lung cancers. J. Clin. Oncol. 17: 2100-2104.

Mori A, Wada H, Nishimura Y, Okamoto T, et al. (2002). Expression of the antiapoptosis gene survivin in human leukemia. Int. J. Hematol. 75: 161-165.

Plate KH, Breier G, Weich HA and Risau W (1992). Vascular endothelial growth factor is a potential tumour angiogenesis factor in human gliomas in vivo. Nature 359: 845-848.

Riedel F, Gotte K, Bergler W and Hormann K (2001). Inverse correlation of apoptotic and angiogenic markers in squamous cell carcinoma of the head and neck. Oncol. Rep. 8: 471-476.

Rohayem J, Diestelkoetter P, Weigle B, Oehmichen A, et al. (2000). Antibody response to the tumor-associated inhibitor of apoptosis protein survivin in cancer patients. Cancer Res. 60: 1815-1817.

Sato K, Sasaki R, Ogura Y, Shimoda N, et al. (1998). Expression of vascular endothelial growth factor gene and its receptor (flt-1) gene in urinary bladder cancer. Tohoku J. Exp. Med. 185: 173-184.

Schultz IJ, Kiemeney LA, Witjes JA, Schalken JA, et al. (2003). Survivin mRNA expression is elevated in malignant urothelial cell carcinomas and predicts time to recurrence. Anticancer Res. 23: 3327-3331. 
Schultz IJ, Kiemeney LA, Karthaus HF, Witjes JA, et al. (2004). Survivin mRNA copy number in bladder washings predicts tumor recurrence in patients with superficial urothelial cell carcinomas. Clin. Chem. 50: 1425-1428.

Smith BD, Smith GL, Carter D, Sasaki CT, et al. (2000). Prognostic significance of vascular endothelial growth factor protein levels in oral and oropharyngeal squamous cell carcinoma. J. Clin. Oncol. 18: 2046-2052.

Suzuki A, Ito T, Kawano H, Hayashida M, et al. (2000). Survivin initiates procaspase 3/p21 complex formation as a result of interaction with Cdk4 to resist Fas-mediated cell death. Oncogene 19: 1346-1353.

Swana HS, Grossman D, Anthony JN, Weiss RM, et al. (1999). Tumor content of the antiapoptosis molecule survivin and recurrence of bladder cancer. N. Engl. J. Med. 341: 452-453.

Sylvester RJ, van der Meijden AP, Oosterlinck W, Witjes JA, et al. (2006). Predicting recurrence and progression in individual patients with stage Ta T1 bladder cancer using EORTC risk tables: a combined analysis of 2596 patients from seven EORTC trials. Eur. Urol. 49: 466-477.

Tran J, Rak J, Sheehan C, Saibil SD, et al. (1999). Marked induction of the IAP family antiapoptotic proteins survivin and XIAP by VEGF in vascular endothelial cells. Biochem. Biophys. Res. Commun. 264: 781-788.

Waters WB (1996). Invasive bladder cancer - where do we go from here? J. Urol. 155: 1910-1911.

Zhu H, Chen XP, Zhang WG, Luo SF, et al. (2005). Expression and significance of new inhibitor of apoptosis protein survivin in hepatocellular carcinoma. World J. Gastroenterol. 11: 3855-3859. 